\title{
TEM Characterization of Self-Assembled Magnetic Nanowires
}

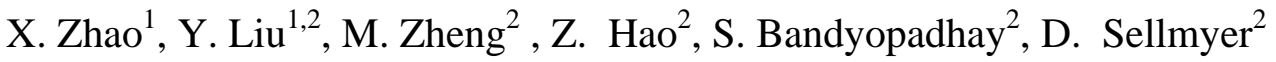 \\ 1 CLAIM, Department of Mechanical Eng. \\ University of Michigan, Ann Arbor, MI 48109-2125 \\ 2 Center for Materials Research and Analysis \\ University of Nebraska, Lincoln, NE 68588.
}

Self-assembled nanowires have the advantage of shape anisotropy and therefore can sustain stable magnetization beyond the superparamagnetic limit of spherical particles. Selfassembled nanowire arrays as a patterned recording medium represent the highest possible recording density for computer hard disks. This paper reports our recent TEM studies of various self-assembled magnetic nanowires using anodic porous alumites. The alumite templates were made by anodizing $\mathrm{Al}$ in $15 \%$ sulfuric acid for 30 minutes, resulting nanoporous structure with diameter around $10 \mathrm{~nm}$ and center to center spacing about $35 \mathrm{~nm}$. Figure 1 shows an example of the ordered pores and released nanowires. The pore diameter can be widened by soaking the anodized aluminum in a $1 \%$ solution of phosphoric acid.

Nanowires of $\mathrm{Ni}, \mathrm{Fe}, \mathrm{Co}, \mathrm{CoFe}$ and $\mathrm{Fe} / \mathrm{Pt}$ were deposited in the template. Details of deposition can be found in [1]. The wires were released by dissolving th e alumina in $0.2 \mathrm{MH}{ }_{2} \mathrm{CrO}_{4} / 0.4 \mathrm{MH}_{3} \mathrm{PO}_{4}$ solution and then picked up by a $\mathrm{Cu}$ grid coated with $\mathrm{C}$ film. JEOL $2010 \mathrm{TEM}$ was used to examine the nanostructure. The $\mathrm{Fe}$ wires and $\mathrm{Ni}$ wires have a relatively larger grain size, with one grain growing across the whole wire, while the Co wires have smaller grain size. Diffraction patterns were taken from all the wires and the ring patterns indicate that all the nanowires are polycrystalline. The crystal structures of the wires were identified by diffraction patterns to $b$ e Fe nanowire: BCC, Co nanowire: mixture of FCC and HCP, Ni nanowire: FCC. While FCC is a metastable phase for bulk Co, it is typically seen in Co nanoparticles or ultra -thin films. Electron diffraction studies indicate the structure of $\mathrm{Fe}_{0.67} \mathrm{Co}_{0.33}$ and $\mathrm{Fe}_{0.93} \mathrm{Co}_{0.07}$ is $\mathrm{BCC}$. The Fe/Pt multilayer is FCC structure. FCC Fe has been observed in as -deposited Fe/Pt multi-layers [2]. The size of the crystallites of Fe, Co and Ni nanowires, as well as the crystalline structure of the Co nanowires depends on dep osition conditions such as the AC frequency, concentration of the solutions, and the chemical treatment of the as-anodized template before deposition. The Fe/Pt multilayer wires have much smaller grain size of about $3 \mathrm{~nm}$ as shown in Figure 2. Pt layers ap pear darker because the higher atomic number with stronger scattering to electrons.

While all the materials in bulk form are soft magnetic materials, significant coercivity enhancement is observed in nanowires of $\mathrm{Co}, \mathrm{Ni}, \mathrm{Fe}$ and $\mathrm{Fe}-\mathrm{Co}$. At room temperature coercivities up to $2.4 \mathrm{kOe}$ in $\mathrm{Co}$ wire, $950 \mathrm{Oe}$ in $\mathrm{Ni}$ wire, $2.4 \mathrm{kOe}$ in $\mathrm{Fe}$ wire, $2.1 \mathrm{kOe}$ in $\mathrm{Fe} / \mathrm{Pt}$ and 2.82.9 kOe in Fe-Co wire are obtained. The remanent magnetization is found to be along the wire axis. The magnetic properties have been explained by the observed nanostructure.

\section{References}

[1] Y. Liu, M. Zhen, H.Zeng and D. J. Sellmyer and S. Bandyopadhyay, In Nanophase and Nanostructured Materials, Eds. Z. L. Wang, Y. Liu and Z. Zhang, Tsinghua Univresity Press and Kluwer Accademic/ Plenum Publishers, Vol. 3, (2001) 210,.

[2] Y. Liu, P. Liu and D.J. Sellmyer, Nanostructured materials, Vol. 12, (1999) 1027.

[3] This research is supported by the US ARO, NSF, NSIC and CMRA at the University of Nebraska-Lincoln. 

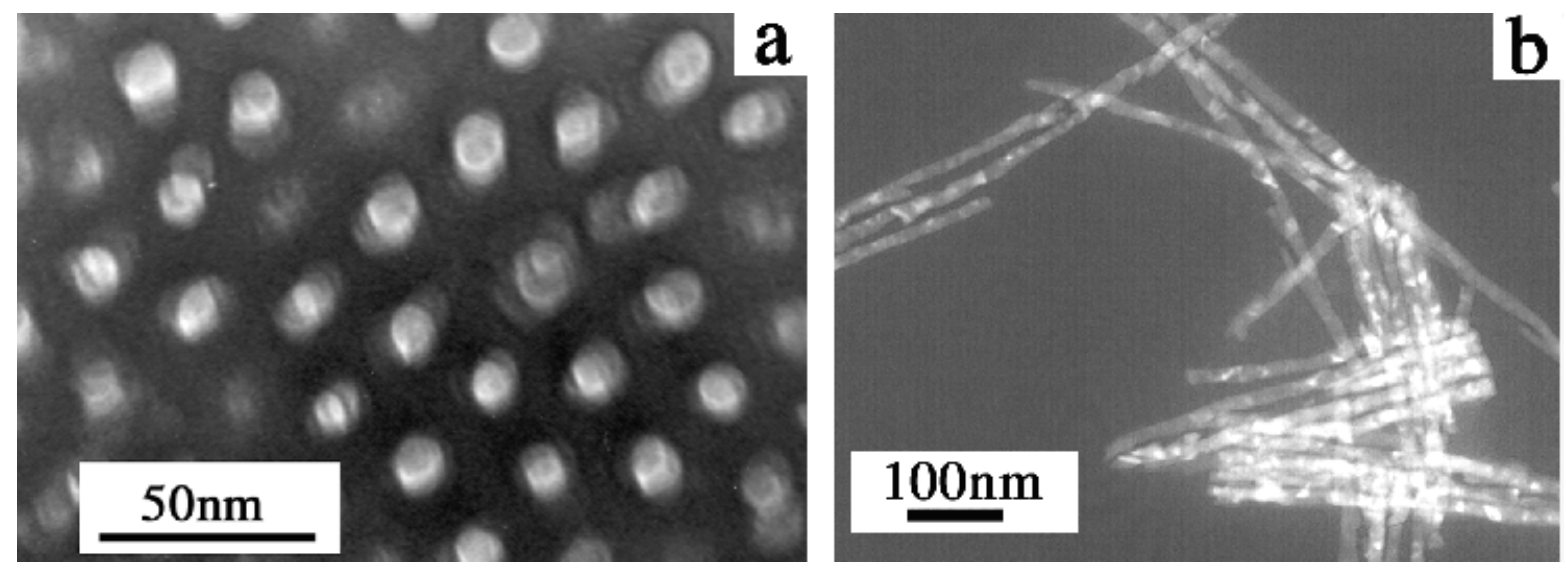

FIG. 1. (a) plane-view of nano-pore template, and (b) Ni nanowires released.

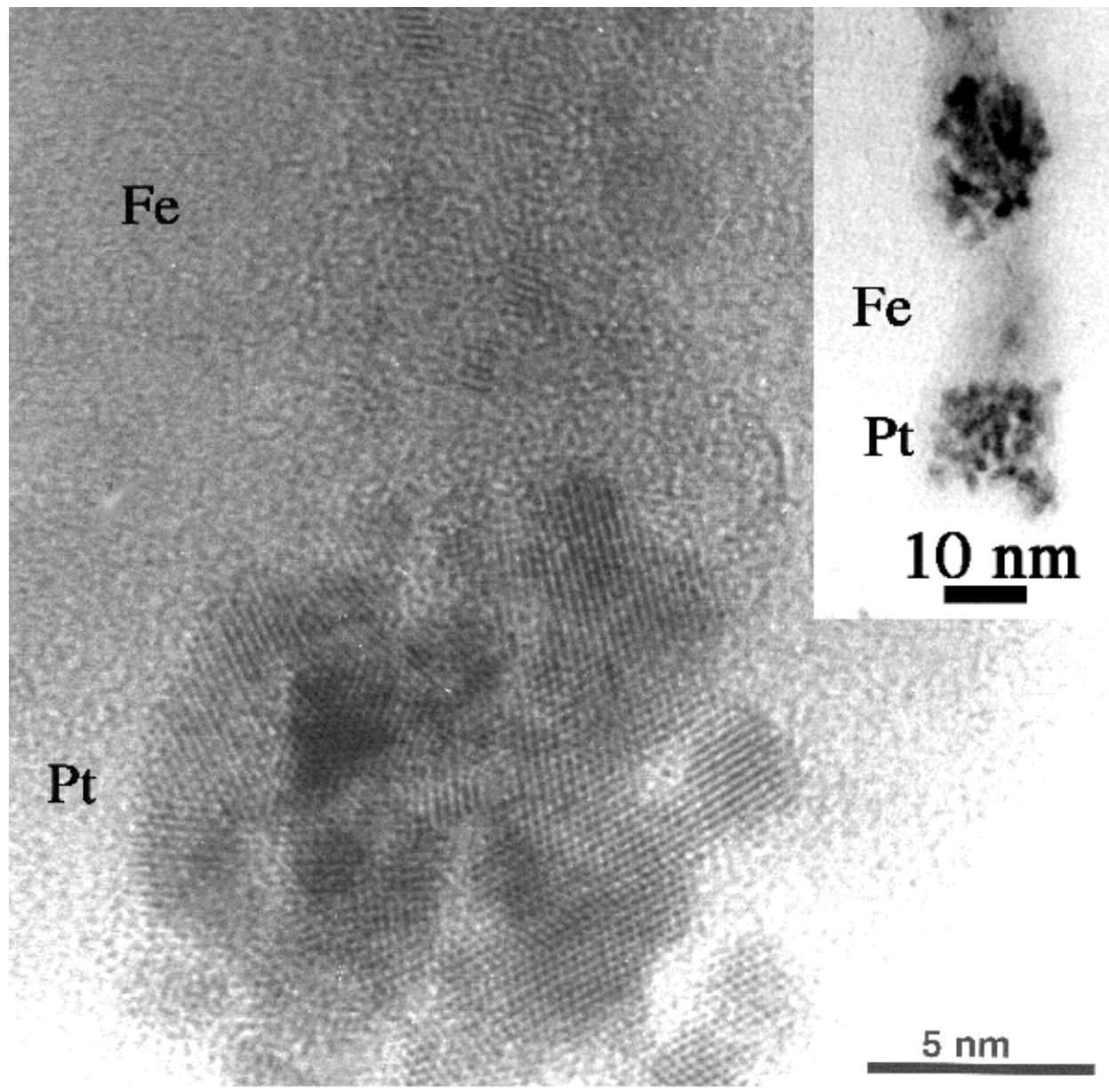

FIG. 2. HRTEM image of Fe/Pt nanowires. 